\title{
Cumulative nonsmoking risk factors increase the probability of developing lung cancer
}

\author{
Carlos E. Bravo-Iñiguez, MD, ${ }^{\mathrm{a}}$ Sam W. Fox, BS, ${ }^{\mathrm{a}}$ Luis E. De Leon, MD, ${ }^{\mathrm{a}}$ Jeffrey N. Tarascio, BA, ${ }^{\mathrm{a}}$
}

Michael T. Jaklitsch, MD, ${ }^{a}$ and Francine L. Jacobson, MD, MPH ${ }^{\mathrm{b}}$

\section{ABSTRACT}

Background: It is estimated that $20 \%$ of lung cancer cases in the United States are among never smokers, yet current screening recommendations only include a small subset of high-risk patients. In this study, 2 models were used to predict the risk of developing lung cancer in subgroups of never smoking patients with additional risk variables.

Methods: The Liverpool Lung Project (LLP) and the Prostate, Lung, Colorectal, and Ovarian Cancer Screening Trial (PLCO) were 2 models used to calculate risk of developing lung cancer. Risk was calculated as a function of age for developing lung cancer within the next 5 to 10 years.

Results: PLCO estimated a peak risk of $16.20 \%$ at age 75 for 30-pack-year smokers with a first-degree relative with lung cancer. LLP estimated a peak risk of $7.3 \%$ over the next 5 years at age 79 for men with 30-pack-year and a first-degree relative with early-onset lung cancer $(<60$ years). Female never smokers with cumulative variables other than smoking had a peak risk of $3.40 \%$ for age 74 to 75 years. In contrast, women with only 30 -pack-year smoking history and no other variable had a peak risk of $2.20 \%$ at age 74 to 75 years.

Conclusions: Models such as LLP and PLCO might be used to identify risk for patients who would otherwise not receive lung cancer screening. These individual risk assessments can be used by patients and providers to assess if one is at substantial risk for developing lung cancer. (J Thorac Cardiovasc Surg $2019 ; 158: 1248-54)$

Comparison of lung cancer risk in 5 years for smoking
and nonsmoking women.
Central Message
Mathematical modeling shows a significant
risk of lung cancer in nonsmoking women
with a family history of lung cancer, environ-
mental exposures, and other nonsmoking risk
factors.
Perspective
Various mathematical models predict the risk
of developing lung cancer using other clinical
variables in addition to smoking history. These
models reveal a surprising cumulative risk in
nonsmoking women.
See Commentaries on page 1255 and
1257 .

The National Lung Screening Trial (NLST) established a $20 \%$ reduction in lung cancer-specific mortality with low-dose computed tomography (LDCT) imaging in 30-pack-year smokers between the ages of 55 and 74 years. ${ }^{1}$ Although smoking remains the predominant risk factor for lung cancer, approximately $15 \%$ to $20 \%$ of all lung cancers occur in never smokers (approximately 33,000-44,000 Americans each year). ${ }^{2}$ Current lung cancer screening

From the ${ }^{\mathrm{a}}$ Division of Thoracic Surgery, and ${ }^{\mathrm{b}}$ Department of Radiology, Brigham and Women's Hospital, Harvard Medical School, Boston, Mass.

Institutional review board approval: Partners 2014P001796, approved September 10, 2014.

Received for publication July 25, 2018; revisions received March 28, 2019; accepted for publication April 20, 2019; available ahead of print June 24, 2019.

Address for reprints: Carlos E. Bravo-Iñiguez, MD, Division of Thoracic Surgery, Department of Surgery, Brigham and Women's Hospital (BWH)/Harvard Medical School (HMS), 75 Francis St, Boston, MA 02115 (E-mail: cbravoiniguez@bwh. harvard.edu).

0022-5223

Copyright $($ C 2019 by The American Association for Thoracic Surgery. Published by Elsevier Inc. This is an open access article under the CC BY-NC-ND license (http:// creativecommons.org/licenses/by-nc-nd/4.0/).

https://doi.org/10.1016/j.jtcvs.2019.04.098 guidelines cover a high-risk cohort of 30-pack-year smokers, yet it offers no screening for never smokers who might have an elevated risk of developing lung cancer. ${ }^{3}$

After publication of the NLST, the National Comprehensive Cancer Network guidelines and the American Association of Thoracic Surgery (AATS) guidelines acknowledged that patients with less than 20 pack-year smoking history and other risk factors might be appropriate for LDCT lung cancer screening. ${ }^{4,5}$ These additive variables include family history, radon exposure, asbestos exposure, and history of pneumonia. We used 2 mathematical risk calculators to explore the additive effect of multiple risk variables in current smokers and never smokers.

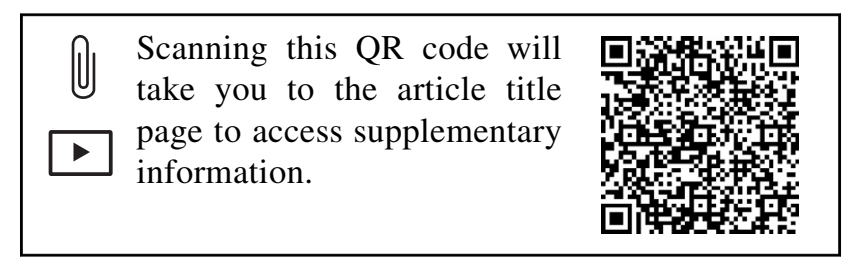




\section{Abbreviations and Acronyms}

AATS $=$ American Association of Thoracic Surgery

AUC = area under the curve

$\mathrm{CI}=$ confidence interval

LDCT $=$ low-dose computed tomography

LLP $=$ Liverpool Lung Project

NLST $=$ National Lung Screening Trial

PLCO $=$ Prostate, Lung, Colorectal, and Ovarian Cancer Screening Trial

In recent years, renewed interest in independent clinical variables that predict the probability of developing lung cancer as a function of age has led to development of several risk prediction models. Two examples of these tools include the Liverpool Lung Project (LLP), which compared risk in 579 lung cancer cases with 1157 age- and sex-matched controls, and The Prostate, Lung, Colorectal, and Ovarian Cancer Screening Trial (PLCO) models derived from comparisons between 38,000 smokers and 70,000 controls. ${ }^{6,7}$

Pretest probability of lung cancer can be estimated using these validated risk prediction tools. Identification of individuals at sufficient risk for lung cancer can refine the current lung cancer screening criteria. For instance, a 5\% risk of developing lung cancer over the next 5 years was chosen as an appropriate benchmark to start lung cancer screening in the United Kingdom Lung Cancer Screening trial. This was a randomized controlled trial in which researchers used a population-based questionnaire to preselect high-risk individuals ( $\geq 5 \%$ over the next 5 years) before randomization to the LDCT or control arm. In this trial, patients with a 30-pack-year smoking history in the 55 to 74 year age range with a less than $5 \%$ risk were excluded from screening, whereas nonsmokers (former or never) with a greater than or equal to $5 \%$ risk were recommended for screening regardless of smoking history. ${ }^{8}$ Such a policy could minimize screening risks (ie, radiation exposure, high false positive rates, and the potential for overdiagnosis), and maximize screening benefit.

Our hypothesis was that these validated risk prediction models which included nonsmokers and never-smokers might identify subpopulations that were appropriate for screening with less than a 30-pack-year smoking history. Specifically, we sought to identify individual risk factors that would significantly increase the risk of lung cancer risk in patients with less tobacco exposure.

\section{METHODS}

\section{The LLP}

The LLP risk model was developed from a case-control study in Liverpool, England. Using a model-based approach, the LLP estimated the individual probability for developing lung cancer within a 5-year period

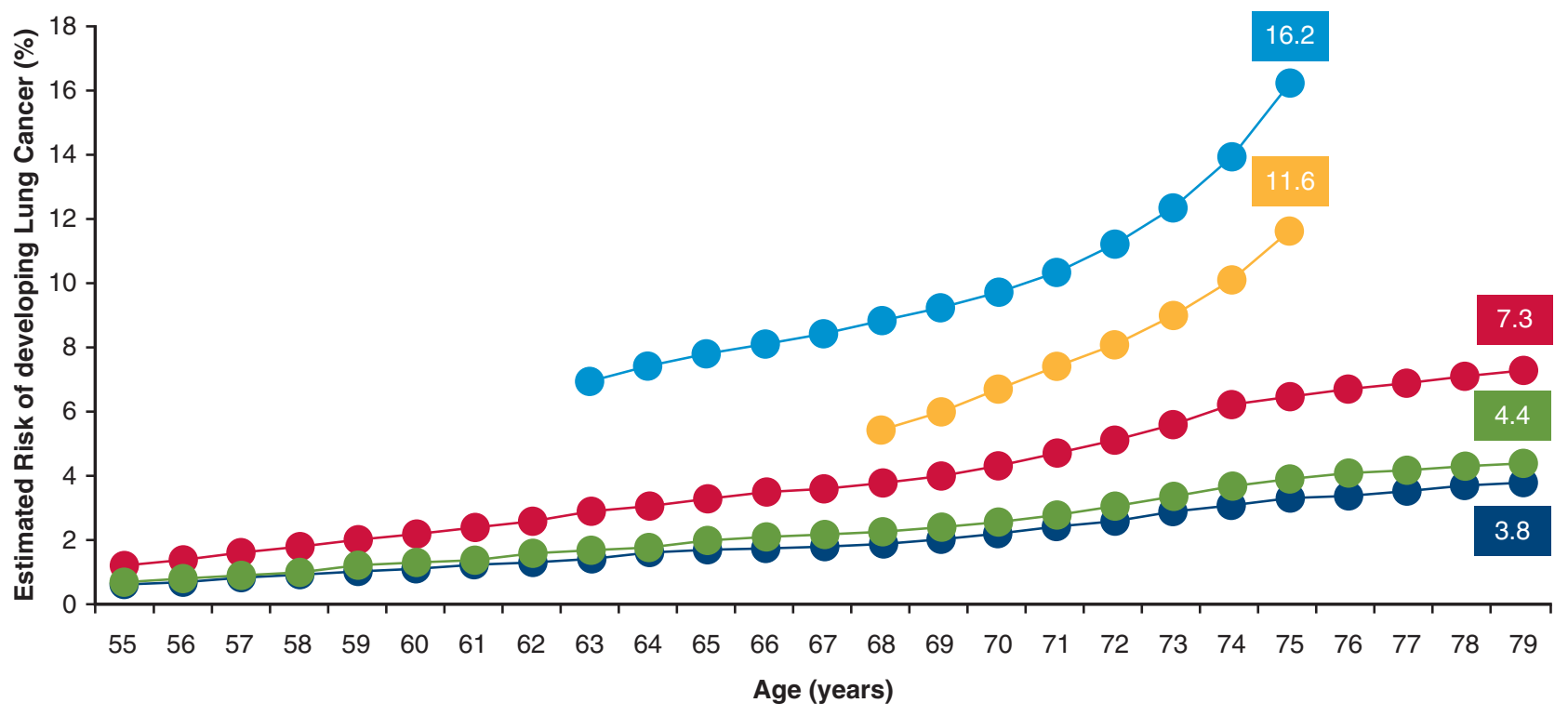

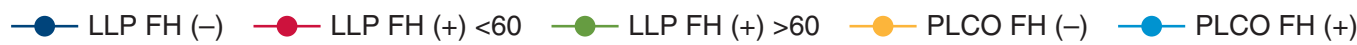

FIGURE 1. Risk of developing lung cancer in men with 30 pack-year smoking history as the only risk factor per Liverpool Lung Project $(L L P)$ and Prostate, Lung, Colorectal, and Ovarian Cancer Screening Trial (PLCO) models. The LLP estimates risk over the next 5 years: (1) LLP with no family history of lung cancer (FH [-]); (2) LLP with a first-degree relative diagnosed with lung cancer before age 60 years $(F H[+]<60)$; and (3) LLP with a first-degree relative diagnosed with lung cancer past age $60(\mathrm{FH}[+]>60)$. PLCO models estimated risk over the next 9 years: (1): PLCO FH [-]; (2) PLCO FH (+); PLCO estimates $<5 \%$ are not displayed. PLCO is not validated for older than 75 years. All profiles were calculated using a fixed body mass index (BMI) of 26, and high-school education. 


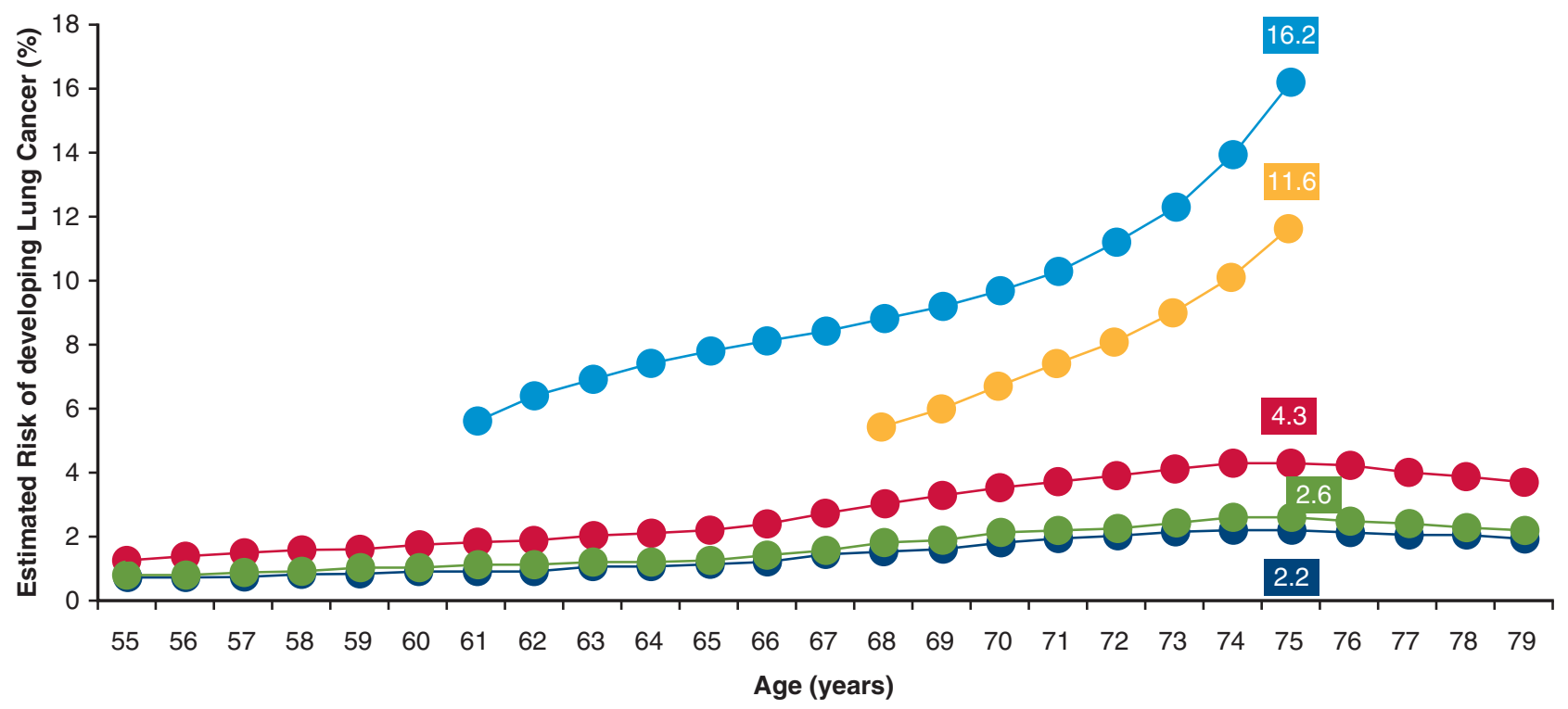

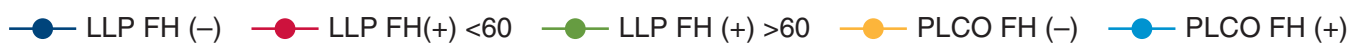

FIGURE 2. Risk of developing lung cancer in women with 30 pack-year smoking history as the only risk factor per Liverpool Lung Project $(L L P)$ and Prostate, Lung, Colorectal, and Ovarian Cancer Screening Trial (PLCO) models. The LLP estimates risk over the next 5 years: (1) LLP with no family history of lung cancer $(F H[-])$; (2) LLP with a first-degree relative diagnosed with lung cancer before age 60 years $(F H[+]<60)$; and $(3)$ LLP with a first-degree relative diagnosed with lung cancer past age $60(\mathrm{FH}[+]>60)$. PLCO models estimated risk over the next 9 years: (1): PLCO FH [-]; (2) PLCO FH (+); PLCO estimates $<5 \%$ are not displayed. PLCO is not validated for older than 75 years. All profiles were calculated using a fixed body mass index $(B M I)$ of 26 , and high-school education.

on the basis of their particular combination of risk factors. Mathematical modeling methods have been published by the authors in the original report. Briefly, data from 579 lung cancer cases and 1157 age- and sex-matched population-based controls within Liverpool were used. Conditional logistic regression models were used to model significant risk factors associated with lung cancer including: smoking history, previous infection (pneumonia), exposure to asbestos, personal history of malignancy, and familial history of lung cancer diagnosed before age 60 years. This multivariable model was combined with age-standardized incident data to estimate absolute risk of developing lung cancer.

The LLP risk model was validated from 3 independent data sets ${ }^{6,9-12}$ : an LLP population-based prospective cohort (area under the curve [AUC], 0.82; 95\% confidence interval [CI], 0.80-0.85), the Harvard case-control study (AUC, 0.76; 95\% CI, 0.75-0.78), and the European Early Lung Cancer case-control study (AUC, 0.67; 95\% CI, 0.64-0.69). The decision utility analysis, which judges the harms and benefits of using a risk model to make clinical decisions, indicated the LLP model performed better than smoking duration or family history alone in stratifying high-risk patients for lung cancer screening.

\section{PLCO Cancer Models}

The PLCO created 2 lung cancer risk models using prospective data from 70,962 control subjects. One risk model was for the general population and a second one was for a subcohort of ever-smokers. Modeling included the following: age, socioeconomic status on the basis of level of education, body mass index, family history of lung cancer, chronic obstructive pulmonary disease, recent chest X-ray, smoking status including never, former, or current smokers, pack-year smoked, and smoking duration. The ever-smokers model also included smoking quit-time (time in years since ever-smokers permanently quit smoking). Logistic regression modeling was used to model significant risk factors.
Internal validation of the PLCO model was carried out by bootstrapping methods using 200 resamples, and external validation cohort was established from the PLCO intervention subjects thought to be cancer-free at the start of the study. For models 1 and 2, bootstrap optimism-corrected receiver operating characteristics curves were 0.86 and 0.80 , and calibration slopes (model-predicted probabilities vs observed probabilities) were 0.99 and 0.98 , respectively. In the external validation sample, models 1 and 2 had AUCs of 0.84 and 0.78 , respectively, with high discrimination in women, men, and white and nonwhite participants. ${ }^{7}$

Available from the LLP investigators is a Web-based tool that facilitates calculation of individual risk, which can be obtained free of charge. ${ }^{13}$ The PLCO model can be obtained as an Excel spreadsheet calculator for the same type of probability estimates. ${ }^{14}$ The AATS has aggregated these 2 models into a single tool for lung cancer risk assessment in an individual, which is publicly available (Video 1$).^{15}$

When completing our risk calculations, we considered the following variables: (1) sex (women and men for both models); (2) age (from 55 to 79 years for LLP and up to 75 years for PLCO because it has not been validated for age older than 75 years); (3) smoking status (all current smokers with 30-pack-year history and never smokers for both models); (4) personal history of cancer (binary, required only for LLP); (5) body mass index (fixed at 26, average in the United States, required for PLCO); (6) education (fixed at high school graduate, average in the United States, required for PLCO $)^{16}$; (7) age of first-degree relative diagnosed with lung cancer ( $<60$ years, $\geq 60$ years, or none, for LLP, binary yes or no for PLCO); (8) environmental factors (yes or no for both models): asbestos, chronic obstructive pulmonary disease, or pneumonia.

Sex and smoking history were fixed while changing one other variable (ie, first-degree relative cancer history) to generate risk plots as a function of age. We then included 3 constant variables (ie, sex, smoking history, and history of previous malignancy) while changing first degree relative cancer history to generate additional plots. 


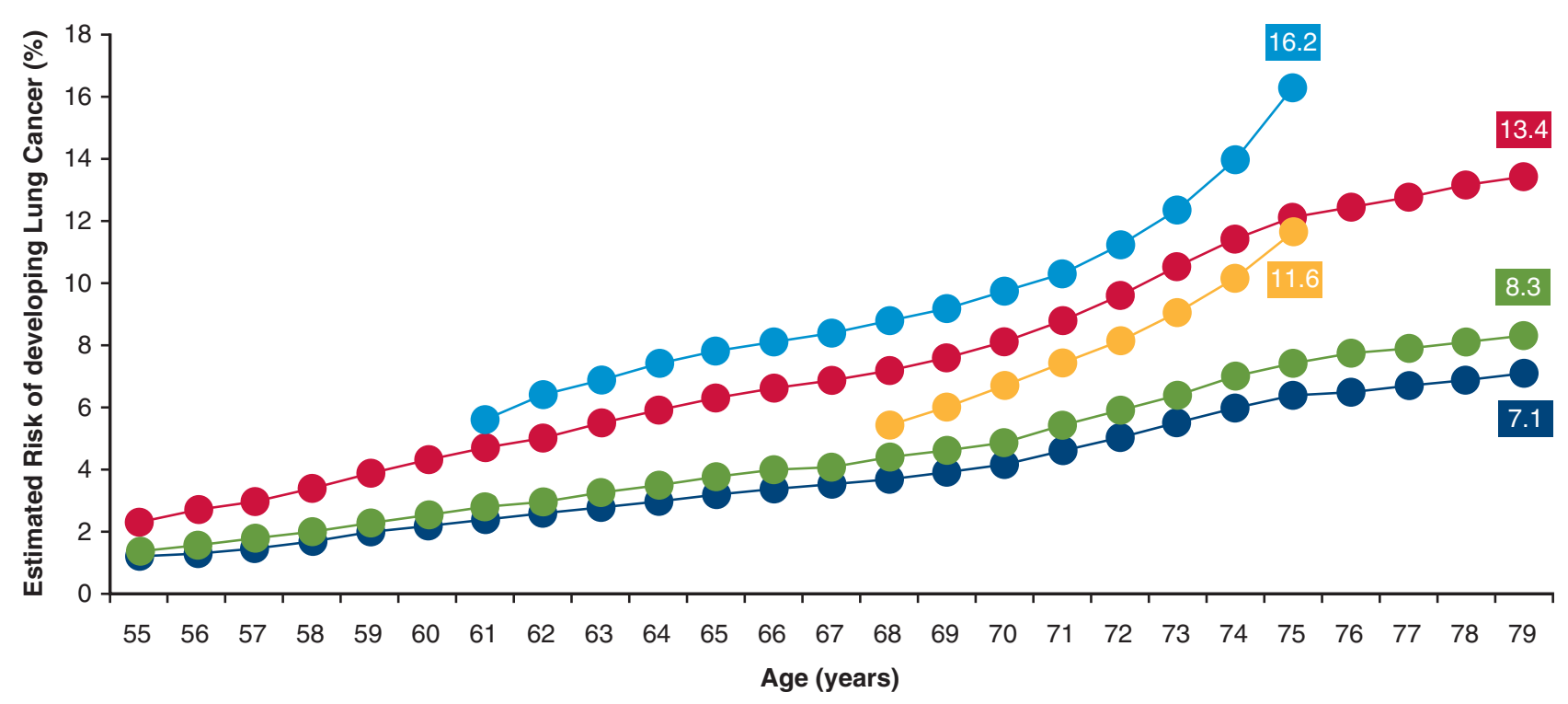

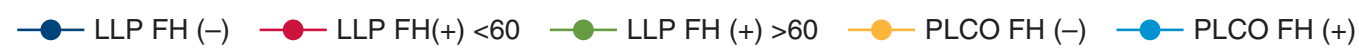

FIGURE 3. Risk of developing lung cancer in men with 30 pack-year smoking history and previous malignancy per Liverpool Lung Project ( $L L P)$ and Prostate, Lung, Colorectal, and Ovarian Cancer Screening Trial (PLCO) models. The LLP estimates risk over the next 5 years: (1) LLP with no family history of lung cancer $(F H[-])$; (2) LLP with a first-degree relative diagnosed with lung cancer before age 60 years $(F H[+]<60)$; and $(3)$ LLP with a first-degree relative diagnosed with lung cancer past age $60(\mathrm{FH}[+]>60)$. PLCO models estimated risk over the next 9 years: (1): PLCO FH [-]; (2) PLCO FH (+); PLCO estimates $<5 \%$ are not displayed. PLCO is not validated for older than 75 years. All profiles were calculated using a fixed body mass index $(B M I)$ of 26 , and high-school education. Previous malignancy refers to individuals who had been previously diagnosed with a malignant tumor.

We plotted risk profiles between 65 and 79 years old and no other comorbidity and compared the estimated risk trend against those in the same age bracket with 30-pack-year smoking history or additional nonsmoking risk factors. To calculate the peak risk, we set all negative variables to the extreme.

\section{RESULTS}

Significant risk with increasing age was predicted by both models in men (Figure 1) and women (Figure 2) with 30-pack-year smoking history. In men (Figure 1) the LLP for 30-pack-year smoking history and no first-degree relatives with lung cancer showed a rise in risk of developing lung cancer in the next 5 years from $0.60 \%$ at age 55 to $3.80 \%$ at age 79 . Furthermore, the LLP model showed an increase in incremental risk as a function of first-degree relative's history of lung cancer. Seventy-nine-year-old men with a 30-pack-year smoking history and a first-degree relative who developed lung cancer at older than the age of 60 years have a $4.40 \%$ risk of lung cancer in the next 5 years. Seventy-nine-year-old men with a 30-pack-year smoking history and a first-degree relative who developed lung cancer at younger than the age of 60 years have a $7.30 \%$ risk over the next 5 years. Risk estimates in the PLCO model are greater. A 75-year-old man with a 30-pack-years smoking history and no first-degree relatives with lung cancer is estimated to have an $11.60 \%$ risk of developing a new lung cancer over 10 years. The same individual with lung cancer in a first-degree relative of any age has a risk of $16.20 \%$ over the next 10 years. Figure 2 shows the same calculations for women. The LLP model has lower risk predictions for women over the next 5 years compared with PLCO (2.20\% for no first-degree relative, $2.60 \%$ for first-degree relative with lung cancer after age 60 years, and $4.3 \%$ for a first-degree relative with lung cancer at younger than age 60 years). The PLCO risk assessments over the next 10 years are the same compared with men. The highest risk was always found in the PLCO model when a patient had a first-degree relative with a history of lung cancer. Regardless of sex and smoking history, the peak risk for this scenario was consistently $16.20 \%$ and found at age 75 years.

We next investigated men (Figure 3) and women (Figure 4) with a 30-pack-year smoking history and a personal history of any malignancy. The LLP model predicted a $7.10 \%$ risk of developing lung cancer within the next 5 years for 75 -year-old men in this category with no first-degree relatives with lung cancer, $8.30 \%$ if a first-degree relative had a history of lung cancer at older than the age of 60 years, and $13.4 \%$ if a first-degree relative had a history of lung cancer at younger than 60 years old. The PLCO model predicted an $11.60 \%$ risk for 75 -yearold men with no family history of lung cancer, and $16.20 \%$ if there was a family history of lung cancer. 


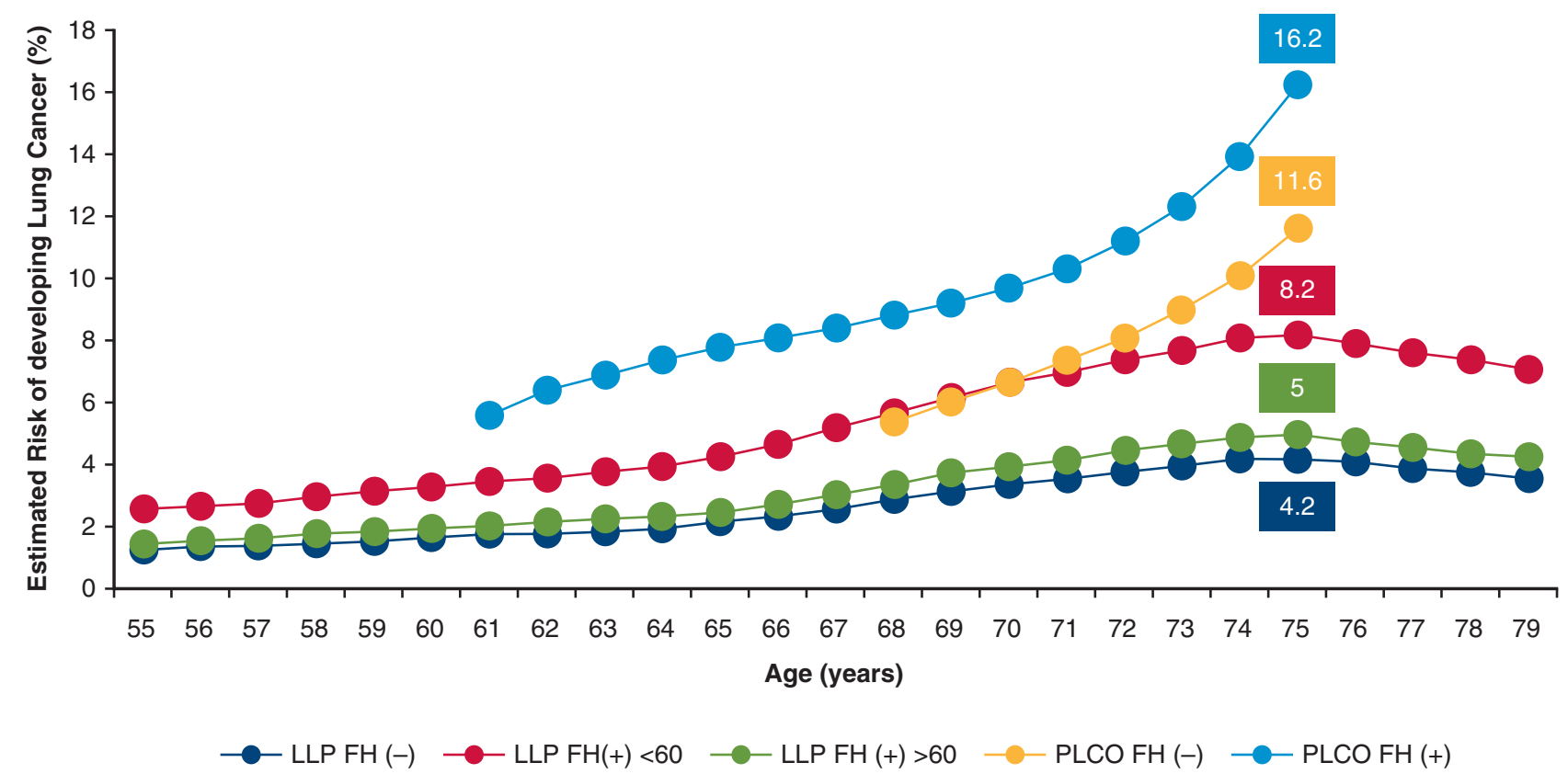

FIGURE 4. Risk of developing lung cancer in women with 30 pack-year smoking history and previous malignancy per Liverpool Lung Project $(L L P)$ and Prostate, Lung, Colorectal, and Ovarian Cancer Screening Trial (PLCO) models. The LLP estimates risk over the next 5 years: (1) LLP with no family history of lung cancer $(F H[-])$; (2) LLP with a first-degree relative diagnosed with lung cancer before age 60 years $(F H[+]<60)$; and $(3)$ LLP with a first-degree relative diagnosed with lung cancer past age $60(\mathrm{FH}[+]>60)$. PLCO models estimated risk over the next 9 years: (1): PLCO FH [-]; (2) PLCO FH (+); PLCO estimates $<5 \%$ are not displayed. PLCO is not validated for older than 75 years. All profiles were calculated using a fixed body mass index $(B M I)$ of 26, and high-school education. Previous malignancy refers to individuals who had been previously diagnosed with a malignant tumor.

The LLP risk model in Figures 1 through 4 also show a slow linear increase in risk over 5 years between ages 55 and 75 years followed by a slow decrease up to age 79 . The PLCO risk model over 9 years shows an exponential increase to age 75 years.

To estimate the maximum risk for a nonsmoking woman of developing lung cancer over the next 5 years, we added the cumulative effects of all other variables and compared this with all favorable risk factors, except 30-pack-year smoking (Figure 5). The highest recorded risk for nonsmoking women with multiple other risk factors approached $7 \%$ over the next 5 years at age 75 years in the LLP model (the only model to estimate risk in never-smokers). This compared with a risk of $<2 \%$ over the next 5 years for women with a 30-pack-year smoking history and no other risk factors. LDCT screening is currently applied to 30-pack-year smoking history women between the ages of 55 to 74 years. Figure 5 also shows the incremental risk of single risk factors for nonsmoking women. This shows that the highest single risk other than tobacco is a first-degree relative with early onset of lung cancer.

\section{DISCUSSION}

There is a common misconception that only heavy smokers develop lung cancer. Current indications for asymptomatic lung cancer screening are only on the basis of age and smoking intensity. However, $25 \%$ of all lung cancer cases within the United States occur in never smokers. ${ }^{1,2}$ Therefore, the development of lung cancer risk prediction models that incorporate individual nontobacco variables is a useful tool. These prediction models can be used to identify other subgroups at high risk for developing lung cancer independent of tobacco history. The emergence of data from the large PLCO and LLP trials provide construction of probability equations of incremental risk and interactions between risk factors for 10 ( 4 for LLP and 6 for PLCO) non-tobacco variables. The validation of these 2 models by experienced teams of statisticians justifies their use for individual risk assessment.

As previously noted, the United Kingdom Lung Cancer Screening Project used a 5\% risk of developing lung cancer over the next 5 years as an appropriate threshold to consider screening a given population. ${ }^{8}$ Of note, Bach et $\mathrm{al}^{17}$ reported the risk of being diagnosed with lung cancer over the next 10 years was $2 \%$ for those who meet the entry criteria of the NLST. ${ }^{2}$ The NLST was a positive randomized trial that showed a $20 \%$ reduction in lung cancer-specific mortality. The NLST has been further supported by the preliminary results of The Dutch-Belgian randomized lung cancer screening trial (NELSON) ${ }^{18}$ which showed a $26 \%$ reduction in male lung cancer deaths over 10 years. ${ }^{19}$ 


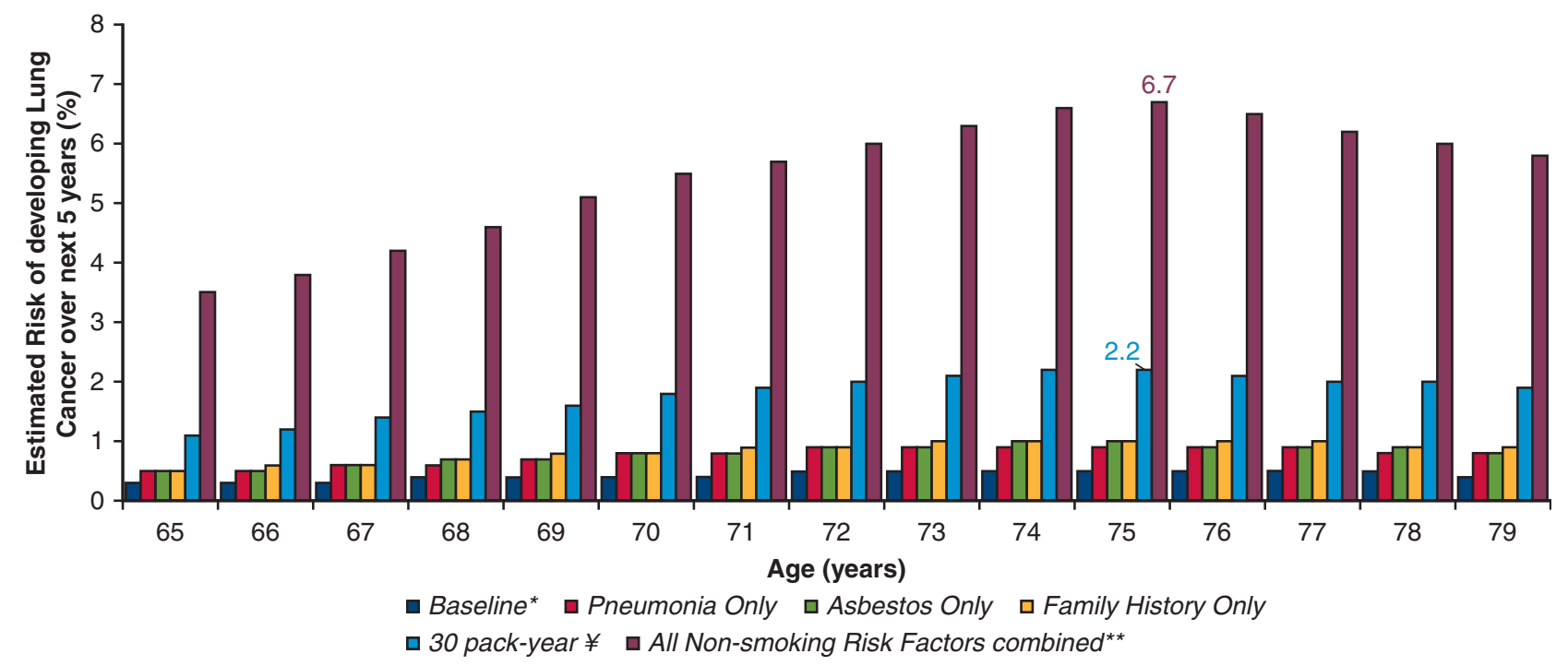

FIGURE 5. Risk differences in women on the basis of combination of risk factors with increasing age. All profiles were calculated using a fixed body mass index of 26, and high-school education*. Baseline (blue bar) represents risk for nonsmoking women with no risk factors for developing lung cancer. Pneumonia only (red bar) represents risk for nonsmoking women with pneumonia as only risk factor. Asbestos only (green bar) represents risk for nonsmoking women with asbestos exposure as only risk factor. Family history (yellow bar) represents risk for nonsmoking women with family history of lung cancer as only risk factor. ${ }^{¥} 30$ pack-year only (cyan bar) indicate risk of lung cancer in next 5 years for women with 30 pack-year smoking history as only risk factor. **Nonsmoking risk factors (mauve bar) show risk of lung cancer in nonsmoking women with all other risk factors, specifically, asbestos exposure, pneumonia, previous malignant tumor, and familial history of lung cancer in a first-degree relative diagnosed before age 60 years.

The graphs in this study revealed a number of nonsmoking subgroups with risk exceeding $5 \%$ over the next 5 years. Therefore, we strongly advocate expanding lung cancer screening for any individual with a calculated risk of $5 \%$ over the next 5 years, and not limit screening to those only with 30-pack-year smoking history.

It is important to note that the only single variable in Figure 5 that raised the incremental risk of lung cancer above $5 \%$ over the next 5 years was a history of 30-pack-year tobacco exposure. The next strongest variable for risk was a first-degree relative developing lung cancer before the age of 60 years. It was the interaction of several variables, excluding tobacco, that was able to raise the incremental risk to exceed $5 \%$ over 5 years. Online risk calculators can facilitate the cumulative effect of multiple variables.

It is possible that lung cancer is continuing to evolve in ways that could change the relative importance of risk factors. These concepts are in line with the disproportionate occurrence of lung cancer in never-smoking women and smokers younger than the age of 65 years. ${ }^{20}$ It is very likely that combinations of smaller risks are represented in never-smokers who develop lung cancer. The order in which risk factors are accrued might also be an important determinant of outcome.

The NLST was a prospective randomized trial that proved a lung cancer-specific survival benefit from LDCT screening. That trial had entry criteria of ages 55 to 74 years, 30-pack-year of tobacco exposure, smoking within the past 15 years, and no competing malignancy. This trial was used to craft public policy by The Centers for Medicare \& Medicaid Services (CMS) and The United States Preventive Services Task Force (USPSTF), a policy that is expected to guide screening for decades. We are suggesting that the limitation of this public policy, as it currently stands, excludes individuals who are predicted to be at high risk for developing lung cancer in the next 5 years, because they do not meet the 30-pack-year smoking exposure criterion. Furthermore, some of these individuals might have a $5 \%$ risk over the next 5 years, or twice the risk of the average participant in the NLST. This study adds credence to the National Comprehensive Cancer Network

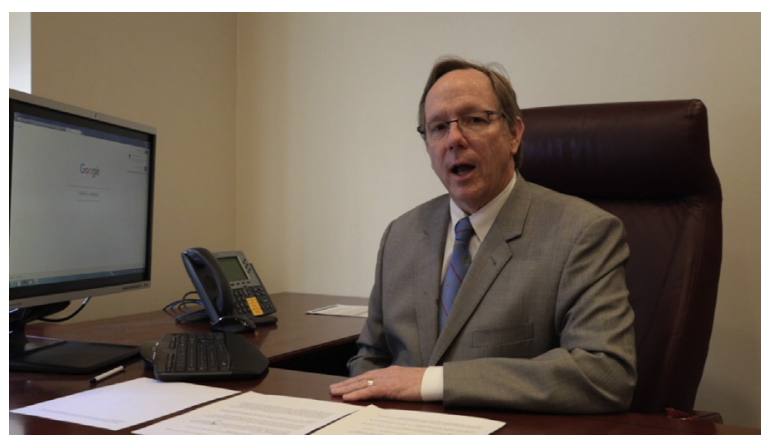

VIDEO 1. Dr Michael Jaklitsch describes the importance of variables beyond tobacco that can increase your risk of developing lung cancer over the next 5 to 9 years using prediction models. Video available at: https://www.jtcvs.org/article/S0022-5223(19)31038-4/fulltext. 
guidelines and the AATS guidelines that both recommended screening for individuals older than the age of 50 years who have 20-pack-years of smoking history and have an additional comorbidity that leads to a cumulative risk of developing lung cancer of $5 \%$ or greater over the next 5 years. 5

Use of the LLP and PLCO mathematical models to predict risk of developing lung cancer in other patients has several limitations. The primary concern for the accurate use of these models is the generalizability and extrapolation to a broader, more diverse cohort. For example, the LLP was developed from a primarily British Caucasian population and validated in a North American subset with similar ethnicities. This might reduce the accuracy when applied to non-Caucasian subjects. Similarly, those who participated in the PLCO model were confined to a narrow age range of 55 to 74 years and were of higher socioeconomic status on average. If applied to the general population with a diversity of ages, educations, and socioeconomic statuses, benefits might be limited. Additionally, in both studies, absolute risk estimates for each combination of risk factors are on the basis of relative risk from the case-controlled studies. Finally, potential predictors such as second-hand smoke, radon, and occupational exposure were excluded from these models and could alter the predictive accuracy. These concerns might be mitigated by using both models together and potentially including other models to obtain a clinically relevant estimation of risk, just as we have done in this analysis. By analyzing the calculations of both models, we have provided a more refined prediction for each individual patient.

\section{CONCLUSIONS}

At this point in time, it is appropriate to use mathematical modeling to predict an individual patient's risk of developing lung cancer and determine if LDCT screening is appropriate for that patient. The future screening methods might depend more heavily on breath tests, genetic tests, and other laboratory predictors. While we await this technology, we should be willing to tailor an individual screening plan for each patient on the basis of these models. The ability to predict lung cancers with the use of these models should result in a more accurate selection of patients to undergo screening, greater cost-effectiveness, reduction of risks, and more lives saved.

\section{Conflict of Interest Statement}

Authors have nothing to disclose with regard to commercial support.

\section{References}

1. National Lung Screening Trial Research Team, Aberle DR, Adams AM, Berg CD, Black WC, Clapp JD, et al. Reduced lung-cancer mortality with low-dose computed tomographic screening. N Engl J Med. 2011;365: 395-409.

2. Sun S, Schiller JH, Gazdar AF. Lung cancer in never smokers-a different disease. Nat Rev Cancer. 2007;7:778-90.

3. U.S. Preventive Services Task Force. Final Update Summary: Lung Cancer: Screening. July 2015. Available at: https://www.uspreventiveservicestaskforce. org/Page/Document/UpdateSummaryFinal/lung-cancer-screening. Accessed June 12, 2019.

4. Wood DE, Eapen GA, Ettinger DS, Hou L, Jackman D, Kazerooni E, et al. Lung cancer screening. J Natl Compr Canc Netw. 2012;10:240-65.

5. Jaklitsch MT, Jacobson FL, Austin JHM, Field JK, Jett JR, Keshavjee S, et al. The American Association for Thoracic Surgery guidelines for lung cancer screening using low-dose computed tomography scans for lung cancer survivors and other high-risk groups. J Thorac Cardiovasc Surg. 2012;144: 33-8.

6. Cassidy A, Myles JP, van Tongeren M, Page RD, Liloglou T, Duffy SW, et al. The LLP risk model: an individual risk prediction model for lung cancer. Br J Cancer. 2008;98:270-6.

7. Tammemagi CM, Pinsky PF, Caporaso NE, Kvale PA, Hocking WG, Church TR, et al. Lung cancer risk prediction: prostate, lung, colorectal and ovarian cancer screening trial models and validation. J Natl Cancer Inst. 2011;103: 1058-68.

8. McRonald FE, Yadegarfar G, Baldwin DR, Devaraj A, Brain KE, Eisen T, et al. The UK lung screen (UKLS): demographic profile of first 88,897 approaches provides recommendations for population screening. Cancer Prev Res (Phila). 2014; 7:362-71.

9. Garcia-Closas M, Kelsey KT, Wiencke JK, Xu X, Wain JC, Christiani DC. A case-control study of cytochrome P450 1A1, glutathione S-transferase M1, cigarette smoking and lung cancer susceptibility (Massachusetts, United States). Cancer Causes Control. 1997;8:544-53.

10. D'Amelio AM, Cassidy A, Asomaning K, Raji OY, Duffy SW, Field JK, et al. Comparison of discriminatory power and accuracy of three lung cancer risk models. Br J Cancer. 2010;103:423-9.

11. Field JK, Liloglou T, Niaz A, Bryan J, Gosney JR, Giles T, et al. EUELC project: a multi-centre, multipurpose study to investigate early stage NSCLC, and to establish a biobank for ongoing collaboration. Eur Respir J. 2009;34: 1477-86.

12. Raji OY, Duffy SW, Agbaje OF, Baker SG, Christiani DC, Cassidy A, et al. Predictive accuracy of the Liverpool lung project risk model for stratifying patients for computed tomography screening for lung cancer: a case-control and cohort validation study. Ann Intern Med. 2012;157:242-50.

13. Roy Castle Lung Cancer Research Programme. My lung risk. Available at: https://secure2.utlnet.co.uk/mylungrisk/welcome.aspx. Accessed June 10, 2019.

14. Brock University. Lung cancer risk calculators. Available at: https://brocku.ca/lungcancer-screening-and-risk-prediction/risk-calculators/. Accessed June 10, 2019.

15. American Association for Thoracic Surgery. Lung cancer risk assessment tool. Available at: https://www.aats.org/aatsimis/AATSWeb/Resources/Lung_Cancer_ Screening/AATSWeb/Association/About/Resources/Lung_Cancer_Risk_Assess ment_Tool.aspx?hkey=29f118a8-d7a6-4bcd-a9b3-7e85484881b8. Accessed June 10, 2019.

16. Ryan C, Bauman K. Educational attainment in the United States: 2015. Available at: https://www.census.gov/data/tables/2018/demo/education-attainment/cpsdetailed-tables.html. Accessed June 10, 2019.

17. Bach PB, Mirkin JN, Oliver TK, Azzoli CG, Berry DA, Brawley OW, et al. Benefits and harms of CT screening for lung cancer: a systematic review. JAMA. 2012;307:2418-29.

18. Horeweg N, van Rosmalen J, Heuvelmans MA, van der Aalst CM, Vliegenthart R, Scholten ET, et al. Lung cancer probability in patients with CT-detected pulmonary nodules: a prespecified analysis of data from the NELSON trial of low-dose CT screening. Lancet Oncol. 2014;15:1332-41.

19. De Koning H, Van Der Aalst C, Ten Haaf K, Oudkerk M. PL02.05. Effects of volume CT lung cancer screening: mortality results of the NELSON randomised-controlled population based trial [abstr]. J Thorac Oncol. 2018; 13(suppl):S185.

20. Mary Horrigan Conners Center for Women's Health and Gender Biology. Out of the shadows: women and lung cancer. Boston, MA: Brigham and Women's Hospital, Harvard Medical School; April 2010.

Key Words: lung cancer, risk, prediction, nonsmoking 
TABLE E1. Liverpool Lung Project multivariate risk model coefficients

\begin{tabular}{|c|c|c|c|c|}
\hline Risk factor & Odds ratio $*(95 \%$ CI $)$ & Odds ratio $\nmid(95 \%$ CI $)$ & $P$ value & Model coefficien \\
\hline Smoking duration, in y & & & $<.001$ & \\
\hline Never & 1.00 (Reference) & 1.00 (Reference) & & 0.000 \\
\hline $1-20$ & $2.48(1.47-4.17)$ & $2.16(1.21-3.85)$ & & 0.769 \\
\hline $21-40$ & $5.81(3.68-9.18)$ & $4.27(2.62-6.94)$ & & 1.452 \\
\hline $41-60$ & $19.24(12.07-30.67)$ & $12.27(7.41-20.30)$ & & 2.507 \\
\hline$>60$ & $41.74(17.86-97.56)$ & $15.25(5.71-40.65)$ & & 2.724 \\
\hline Previous diagnosis of pneumonia & & & .002 & \\
\hline No & 1.00 (Reference) & 1.00 (Reference) & & 0 \\
\hline Yes & $1.62(1.21-2.17)$ & $1.83(1.26-2.64)$ & & 0.602 \\
\hline Exposure to asbestos & & & $<.001$ & \\
\hline No & 1.00 (Reference) & 1.00 (Reference) & & 0 \\
\hline Yes & $1.94(1.46-2.59)$ & $1.89(1.35-2.62)$ & & 0.634 \\
\hline Previous diagnosis of malignant tumor & & & .005 & \\
\hline No & 1.00 (Reference) & 1.00 (Reference) & & 0 \\
\hline Yes & $2.55(1.76-3.71)$ & $1.96(1.22-3.14)$ & & 0.675 \\
\hline Family history of lung cancer & & & .01 & \\
\hline No & 1.00 (Reference) & 1.00 (Reference) & & 0.000 \\
\hline First-degree relative (diagnosis $<60 \mathrm{y}$ ) & $1.54(1.03-2.29)$ & $2.02(1.18-3.45)$ & & 0.703 \\
\hline First-degree relative (diagnosis $>60 \mathrm{y}$ ) & $1.08(0.80-1.46)$ & $1.18(0.79-1.76)$ & & 0.168 \\
\hline
\end{tabular}

CI, Confidence interval. *Odds ratios derived from univariate conditional logistic regression. $\dagger$ Odds ratios derived from multivariate conditional logistic regression. Adapted from Cassidy et al. ${ }^{6}$

TABLE E2. Logistic regression lung cancer prediction models prepared in all of the PLCO control arms (model 1) and in smokers only (model 2)

\begin{tabular}{|c|c|c|c|c|}
\hline \multirow[b]{3}{*}{ Variable } & \multicolumn{2}{|c|}{ Model 1} & \multicolumn{2}{|c|}{ Model 2} \\
\hline & \multicolumn{2}{|c|}{ All PLCO control arm* } & \multicolumn{2}{|c|}{ Smokers only in PLCO } \\
\hline & OR $(95 \%$ CI $)$ & $P$ value & OR $(95 \%$ CI $)$ & $P$ value \\
\hline \multicolumn{5}{|l|}{ Age, per y } \\
\hline Age spline 1 & $1.212(1.110-1.322)$ & $<.001$ & $1.245(1.130-1.372)$ & $<.001$ \\
\hline Age spline 2 & $0.732(0.551-0.972)$ & .031 & $0.705(0.505-0.984)$ & .040 \\
\hline Age spline 3 & $1.884(0.917-3.869)$ & .085 & $2.205(0.860-5.651)$ & .100 \\
\hline Education, per 1 of 7 levels change & $0.930(0.890-0.971)$ & .001 & $0.928(0.887-0.971)$ & .001 \\
\hline BMI, per 1-unit change & $0.970(0.955-0.985)$ & $<.001$ & $0.972(0.956-0.988)$ & .001 \\
\hline Family history of lung cancer, yes vs no & $1.564(1.323-1.848)$ & $<.001$ & $1.561(1.313-1.856)$ & $<.001$ \\
\hline COPD, yes vs no & $1.380(1.153-1.651)$ & $<.001$ & $1.374(1.145-1.648)$ & .001 \\
\hline Chest X-ray in past 3 ys, per 1 of 3 levels & $1.114(1.020-1.217)$ & .017 & $1.117(1.019-1.225)$ & .019 \\
\hline \multicolumn{5}{|l|}{ PKYR, per 1 pack-year } \\
\hline PKYR spline 1 & $1.108(1.073-1.144)$ & $<.001$ & $1.059(1.044-1.074)$ & $<.001$ \\
\hline PKYR spline 2 & $0.500(0.392-0.636)$ & $<.001$ & $0.949(0.935-0.964)$ & $<.001$ \\
\hline \multicolumn{5}{|l|}{ Smoking duration, linear, per $1 \mathrm{y}$} \\
\hline Duration spline 1 & $0.986(0.949-1.025)$ & .480 & & \\
\hline Duration spline 2 & $1.127(1.019-1.246)$ & .020 & & \\
\hline Duration spline 1 & $0.986(0.949-1.025)$ & .480 & & \\
\hline \multicolumn{5}{|l|}{ Smoking quit-time in smokers, per $1 \mathrm{y}$} \\
\hline Quit-time spline 1 & & & $0.945(0.918-0.974)$ & $<.001$ \\
\hline Quit-time spline 2 & & & $1.047(1.011-1.085)$ & .010 \\
\hline \multicolumn{5}{|l|}{ Smoking status } \\
\hline Never/former & Baseline & $<.001$ & Baseline & .010 \\
\hline Current & $1.721(1.426-2.077)$ & & $1.356(1.077-1.708)$ & \\
\hline
\end{tabular}

PLCO, Prostate, Lung, Colorectal, and Ovarian Cancer Screening Trial; $O R$, odds ratio; $C I$, confidence interval; $B M I$, body mass index; $C O P D$, chronic obstructive pulmonary disease; PKYR, pack-years smoked. *Splines for age, PKYR, and smoking duration in model 1 are on the basis of all PLCO control subjects. Knots for age were at 55, 60, 65, and 72 years. Knots for pack-years were at 0, 2.25, and 49 pack-years. Knots for smoking duration were at 0,6 , and 41 years. $†$ Splines for age, PKYR, and quit-time in model 2 are on the basis of the distribution of these variables in smokers only. Knots for age were at 55, 60, 64, and 72 years. Knots for pack-years were at 3.25, 23.25 , and 63 pack-years. Knots for quit-time were at 0,15 , and 35 years. Adapted from Tammemagi et al. ${ }^{7}$ 